\title{
Mapeo del conocimiento para la enseñanza interdisciplinaria en ingeniería
}

Freddy Marín-González ${ }^{1}$, John Grimaldo-Guerrero ${ }^{2}$, Manuel Mendoza-Becerra ${ }^{3}$, y Alexa Senior-Naveda ${ }^{1}$ (1) Departamento de Humanidades, Universidad de la Costa, Atlántico - Colombia (correo-e: fmarin1@cuc.edu.co; asenior@cuc.edu.co)

(2) Departamento de Energía, Universidad de la Costa, Atlántico - Colombia (e-mail: jgrimald1@cuc.edu.co)

(3) Departamento de Matemáticas y Estadística, Universidad del Norte, Atlántico - Colombia

(correo-e: mabecerra@uninorte.edu.co)

Recibido Mar. 5, 2020; Aceptado May. 6, 2020; Versión final Jul. 14, 2020, Publicado Dic. 2020

\section{Resumen}

En este estudio se analiza la pertinencia conceptual y metodológica del mapeo de conocimiento para la enseñanza interdisciplinaria de conceptos estructurantes entre ingeniería, educación y ciencias básicas. El diseño implica la aplicación y validación de las etapas de ciclo de mejora continua (the "plan-do-check-act", PDCA), donde se inserta el mapeo de conocimiento como estrategia para fortalecer el pensamiento de base interdisciplinar en estudiantes de ingeniería eléctrica con el tema generador: sistema de potencias. Los resultados evidencian que la enseñanza integrada de estas disciplinas, desde un enfoque constructivista, puede fortalecerse con los mapas de conocimiento de naturaleza conceptual, contribuyendo al mejoramiento del desempeño académico y calidad del proceso. Se concluye que los estudiantes mediante la representación gráfica confieren significados a la red de relaciones conceptuales emergente, y para el docente es una guía de los diferentes itinerarios de mediación, a través de los cuales construye un conocimiento integrador.

Palabras clave: mapeo de conocimiento; enseñanza interdisciplinaria; práctica docente; mediación didáctica

\section{Knowledge mapping for interdisciplinary engineering teaching}

\begin{abstract}
The primary objective of the present study is to analyze the conceptual and methodological relevance of knowledge mapping for interdisciplinary teaching of structuring concepts in engineering, education, and basic sciences. The design implies the application and validation of the continuous improvement cycle, the plan-docheck-act (PDCA), where knowledge mapping was inserted as a strategy to strengthen interdisciplinary core thinking about power systems in electrical engineering students. The results show that the integrated teaching of these disciplines, from a constructivist approach, can be strengthened with knowledge maps of conceptual nature, contributing to the improvement of academic performance and process quality. It is concluded that students confer meanings to the network of emerging conceptual relationships through graphic representation. In addition, this is a guide for the instructor about the different mediation itineraries by which she/he builds an integrative knowledge.
\end{abstract}




\section{INTRODUCCIÓN}

La formación integral en programas académicos de ingeniería se concibe como un proceso dinámico donde se generan espacios cognitivos de integración entre los saberes propios de la disciplina, con los aportes que devienen de otras áreas del conocimiento, de forma tal que se configuran espacios didácticos de convergencia que se hacen significativos y pertinentes en el ámbito de un currículo contextualizado y emergente. Desde esta perspectiva se visualiza un enfoque constructivista en la formación integral del ingeniero, cuyo fundamento teórico ha sido validado en atención a los fundamentos de Vygotsky (1997) y Ausubel (1983) cuando destacan la importancia de que el sujeto configure su propia estructura cognitiva. Se infieren aportes desde la psicología a la comprensión de la didáctica disciplinar, tal es el caso de Piaget (1970) que aun cuando sus principales postulados no se corresponden directamente con el proceso de enseñanza, puede asociarse con condiciones de contexto para la producción didáctica.

El referido enfoque constructivista evidencia una concepción de práctica pedagógica que resulta en procesos de mediación y trasposición didáctica. Ello traduce a juicio de Chevallard (1985) la necesidad de transformar el saber científico original, en un objeto de enseñanza de fácil comprensión, en correspondencia con los significados que el estudiante le confiere; más concretamente refiere un proceso de cambio, evolución y desarrollo de los contenidos propios de la ingeniería, para que adquieran su lógica en atención a su andamiaje cognitivo. Ello supone, tal como lo sostiene Cabero y Barroso (2016) que los procesos formativos deben integrar el dominio de los contenidos de carácter tecnológico a los procesos formativos; en este sentido, el dominio del docente, tanto de su disciplina, como de la estrategia de mediación didáctica - pedagógica resulta clave para fortalecer el perfil de competencias en sus estudiantes.

Desde una visión innovadora la enseñanza de la ingeniería concibe al estudiante como sujeto activo en la construcción de un aprendizaje significativo, cuyos referentes consideran aspectos de carácter cognitivo, procedimental e incorpora sus vivencias y experiencias. Tal como señala Ausubel (1983) el aprendizaje significativo conlleva procesos de organización y sistematización de la información obtenida confiriendo significados que permiten al individuo una mejor descripción, explicación o comprensión de la realidad que traduce la construcción de un conocimiento que encuentra sentido en función de la realidad o contexto. Consecuentemente, el aprendizaje significativo expresa desarrollo de competencias asociadas a la formación integral del estudiante. Al respecto, autores como (Tobón, 2006; Albert et al.,2017; Marín et al., 2018) destacan que los perfiles profesionales por competencias derivan en estándares de desempeño definidos en función de principios como la contextualización, idoneidad e integralidad.

En atención a lo expuesto el presente artículo se contextualiza en la concepción de una didáctica interdisciplinaria, como categoría que fundamenta el aprendizaje significativo entre ingeniería y disciplinas asociadas. Se aborda la integración científica mediante la relación entre diferentes dominios cognitivos; para visualizar lo descrito, en la presente investigación se refieren contenidos de ingeniería eléctrica en conjunción con la matemática, física, didáctica, pedagogía y currículo. A decir de Pelc (2002) en procesos de integración científica-tecnológica surgen los conceptos estructurantes y temáticas generadoras como constructos de relación convergente entre los aportes de las diferentes disciplinas que sirven de insumo para que el individuo construya nuevos conocimientos sobre la base de la asignación de significados a los datos e información procesada.

En este artículo, se construyen espacios de convergencia alrededor de un núcleo temático generador (Sistemas de potencia), con la disposición de conceptos estructurantes, entre los que destacan en ingeniería: sistemas por unidad, matriz de admitancia, diagrama de secuencias, matriz de fallas; por su parte en ciencias básicas se consideran las leyes de Ohm y Kirchhoff, números complejos; la enseñanza integrada de los referidos constructos se operacionaliza a través de la transposición y mediación didáctica, resolución de problemas, formación integral por competencias, práctica pedagógica y perfil del docente, desarrollo del pensamiento lógico, formal y crítico.

En este sentido, la enseñanza de la ingeniería requiere de aproximaciones metodológicas para orientar la construcción de un conocimiento de base interdisciplinar. Destacan los mapas de conocimiento propuestos por Pelc (2002) como representaciones gráficas, que aun cuando los concibe para la gestión tecnológica, en esta investigación por procesos de transferencia y analogía resultan pertinentes en la mediación didáctica, por cuanto contribuyen al desarrollo de competencias en la aprehensión de fenómenos, situaciones problemas y contenidos temáticos, cuya complejidad requiere un abordaje que trasciende la óptica disciplinar y se ubica en el plano de la integración convergente entre ciencias complementarias.

Desde este contexto referencial el propósito de la investigación es analizar la pertinencia conceptual y metodológica del mapeo de conocimiento para la enseñanza integrada e interdisciplinaria de conceptos estructurantes entre Ingeniería, Educación y Ciencias Básicas; se formula como interrogante: ¿Cuáles serán 
las vías aproximativas para construir conocimiento interdisciplinario en el proceso de enseñanza de la Ingeniería, a través del mapeo de conocimiento como metodología que permite la integración de conceptos estructurantes, propios de sus dominios teóricos y la convergencia de otras disciplinas, como las Ciencias Educacionales y Ciencias Básicas?. El diseño implica la aplicación y validación de las etapas de ciclo de mejora continua (PDCA) propuesto por Shewhart y Deming (1939) donde se inserta el mapeo de conocimiento como estrategia para fortalecer el pensamiento interdisciplinario en estudiantes de ingeniería eléctrica, con el tema generador: sistema de potencias.

\section{OTROS ANTECEDENTES}

La enseñanza de las disciplinas científicas, entre las que puede referirse la ingeniería, no debe concebirse de forma aislada o fragmentada; se demandan herramientas de orden intelectual que permitan consolidar una visión integradora, un todo articulado, donde se trascienda una óptica monodisciplinar y se generen espacios de interacción mediante flujos de información y conocimiento. La concepción de la formación en ingeniería como territorio de conocimiento integrador y convergente deriva en la comprensión amplia de sus problemas, situaciones o fenómenos en contraposición a una visión reduccionista o por estancos de la realidad emergente, lo que autores como (Van der Linde, 2007; Guzmán et al., 2019) definen como una articulación interdisciplinar fundamentada en principios de cooperación. Al respecto, Palomares y Chisvert (2016) destacan la importancia del aprendizaje cooperativo, como proceso que permite generar capacidades para construir conocimiento, privilegiando sus dimensiones conceptuales, procedimentales y actitudinales.

En la enseñanza interdisciplinaria de la ingeniería se atiende una perspectiva dialógica y dialéctica. Autores como Castro y Li (2014) apuestan por la creación de un lenguaje común que conlleve a la construcción de isomorfismos conceptuales y lingüísticos como fundamento de un metalenguaje; se orienta un conocimiento integrador de base conceptual y metodológica para la interpretación y resolución de los problemas propios del área estudiada. Consecuentemente, la enseñanza de la ingeniería adquiere significativas implicaciones en el ámbito de paradigmas emergentes, que centran la discusión en cómo trascender de un ciclo didáctico memorístico, unidireccional, orientado más al producto que a los procesos, a experiencias de aprendizaje donde se incorporen estrategias de mediación fundamentadas en el desarrollo de un pensamiento sistémico, integrador y de perspectiva reticular.

Para Cervantes et al. (2016) la concepción reticular en la enseñanza interdisciplinaria permite la conformación de nodos de interacción entre los aportes de las diferentes disciplinas, como una vía para generar capacidades individuales y colectivas donde se orienten procesos de creación e innovación científico técnica, lo que (Bravo et al.,2013; Espinoza y Marín, 2019) enuncian como cultura reticular. En la dinámica de redes los actores abstraen la realidad, para establecer un plano ontológico de base interdisciplinar que expresa la naturaleza cognitiva y metacognitiva de la representación configurada. En este sentido Rodríguez (2009) concibe los procesos interdisciplinarios en ciencias en dos direccionalidades, por un lado posibilitan la construcción de una relación dialógica y dialéctica en torno al conocimiento, fundamentos teóricos conceptuales y su episteme como forma de comprender percepciones, tensiones y dinámicas inherentes a dichas interacciones, y por el otro permiten generar flujos de convergencia o complementariedad en atención a conceptos, métodos, técnicas de trabajo, que en espacios de integración didáctica dinamicen la práctica pedagógica, contribuyendo a la resignificación del currículo en los programas académicos de formación, tanto en ciencias básicas como ingeniería.

A decir de Quarless y Nieto (2013) la construcción del conocimiento en ciencias implica el desarrollo de competencias y habilidades para procesar información. Resulta significativo que el estudiante configure representaciones mentales múltiples de base cognitiva; por tanto, los niveles de comprensión suponen destrezas para visualizar esquemas relacionales, así como también, reconocer e interpretar formulas y ecuaciones propias de las ciencias. Los autores destacan que un razonamiento cuantitativo incluye capacidades de integración para las relaciones numéricas, desde una dimensión conceptual o dominio científico; el plano de abstracción conlleva procesos de análisis y evaluación de los contenidos integrados.

Específicamente en la ingeniería, se enfatiza la necesidad de revisar los currículos, métodos de enseñanza y la gestión instruccional para fortalecer una didáctica interdisciplinaria y de integración. Estos requerimientos se han orientado en las últimas décadas, tal como lo señala la Organización de las Naciones Unidas para la Educación, la Ciencia y la Cultura (UNESCO, 2012) a la reconstrucción de los planes de estudio con fundamento en la mediación innovadora, de forma tal que en lo conceptual y operativo se trascienda el dominio cognitivo disciplinar. Ello supone el desarrollo de competencias para participar de la generación, transferencia y compartición del conocimiento interdisciplinar, desde la comunicación, diálogo, reflexión independiente, trabajo en equipo, análisis crítico y creativo de la realidad propia del área de estudio. En este ámbito emergen otras corrientes educativas asociadas a la enseñanza de la ciencia, tecnología, ingeniería y matemáticas (STEM: Science. Technology-Engineering-Mathematics), que apoyan los argumentos precedentes y 
contribuyen al desarrollo del pensamiento crítico, mediante la aplicación de distintas vías de solución a un problema con la disposición de la menor cantidad de recursos. (Kennedy y Odell, 2014; Domínguez et al., 2019). Consecuentemente, se infieren importantes grados de independencia cognitiva con experiencias de aprendizaje que contribuyan a la formación integral.

Para Castro y Li (2014) destaca en la práctica pedagógica el conocimiento que manejan los docentes para identificar los posibles errores en la abstracción de conceptos por parte del estudiante; se infiere como determinante la estrategia aplicada para que el aprendiz sea consciente de la valoración del constructo en la aprehensión de las temáticas disciplinares y sus mecanismos de integración. Autores como (Coll y Engel, 2014; Robles et al., 2017) sostienen la importancia de que el estudiante participe de una formación integral de sus capacidades, para la creación e innovación en el dominio de contenidos; se requiere una visión crítica en correspondencia a sus intereses, necesidades, propósitos personales; en síntesis, prevalecen procesos continuos de autoaprendizaje que conducen la toma de decisiones, en relación a las condiciones, formas y mecanismos que emplea en la construcción de un conocimiento significativo.

Consecuentemente, la construcción de conocimiento fundamentado en la noción de cambio conceptual y aprendizaje significativo se concibe en una visión sistémica e interdisciplinaria, por cuanto la realidad donde coexisten los problemas propios de la ingeniería y sus dominios complementarios no puede ser estudiada desde una única perspectiva. Para Steffens et al. (2017) el enfoque interdisciplinar en la didáctica expresa uno de sus principales aportes mediante la contribución al desarrollo del pensamiento crítico en los estudiantes. Desarrollan competencias para categorizar, analizar, sintetizar y valorar información, discriminando sus grados de aplicación en función de su utilidad, generando las debidas inferencias argumentativas. Transfiriendo al ámbito didáctico-curricular los aportes de Verloop et al. (2001) la interdisciplinariedad representa en sí misma una estrategia pedagógica que implica la interacción de varias disciplinas, mediante relaciones colaborativas y dialógicas que inducen la emergencia de un nuevo conocimiento. La referida práctica supone la concepción de isomorfismos lingüísticos que permiten la convergencia disciplinar, al hacer más difusas las fronteras entre sus dominios fundamentales.

En el caso particular de la formación en ingeniería es necesario fortalecer un perfil centrado en competencias que orienten el desarrollo de potencialidades de carácter técnico-científico a la luz de la propia disciplina; para (Ramírez et al., 2016; Albert et al., 2017) la especialización disciplinar en ingeniería se expresa a través de la concepción, diseño, implementación y evaluación de sistemas complejos, que pueden ser tanto abstractos, como empíricos, para ello se requiere el fortalecimiento del pensamiento lógico formal y la aprehensión propositiva del contexto donde se produce el conocimiento. Para desarrollar estas competencias es preciso concebir la didáctica de la ingeniería como un territorio de conocimiento integrado e interdisciplinario, en el cual convergen conceptos, técnicas, procesos del área, y de otras ciencias complementarias, como las ciencias básicas y la educación.

Este enfoque supone el desarrollo de competencias comunicativas y ciudadanas centradas en el trabajo en equipo y los mecanismos de autoaprendizaje. El desarrollo de competencias en los estudiantes de programas académicos de ingeniería conlleva procesos reflexivos de alta complejidad y abstracción; se configuran representaciones mentales desde mecanismos de selección, codificación, diferenciación progresiva, descripción, categorización, análisis, interpretación, en atención a la red de relaciones que fundamentan el cambio conceptual. Según De Bono (1992) se privilegian estilos y formas de pensamiento divergente o creativo que describen una dinámica espontánea de ideas generadas de un modo cognitivo emergente, las cuales expresan diferentes vías aproximativas para la solución de una situación problema.

Se infiere un sentido amplio en la concepción de competencia interdisciplinaria, asociada al perfil de desempeño, cuando el individuo participa propositivamente en la transformación del objeto de estudio, referido a las situaciones problemas. El desarrollo metacognitivo del sujeto se expresa en su capacidad de actuación para la aprehensión y transformación del fenómeno. Para Follari (2007) en un intento por flexibilizar y ampliar los marcos de referencia de la realidad emergen espacios cognitivos de base interdisciplinaria. Concretamente en ingeniería se incorporan a la mediación didáctica, técnicas de trabajo que inducen la metacognición. Tal es el caso del mapeo de conocimiento. La didáctica de la ingeniería fundamentada en la configuración de mapas de conocimiento de naturaleza conceptual supone el despliegue de capacidades asociadas a la necesidad por desarrollar estilos de pensamiento de base interdisciplinaria y de naturaleza sistémica. Desde este referente se concibe una estrategia de mediación donde los conceptos, proposiciones, teorías y otros eventos definen relaciones de convergencia e interdependencia, cuya visual permite aproximarse a un tejido reticular representativo de la información disponible.

De acuerdo con Pelc (2002) el mapeo de conocimiento como proceso permite identificar los diferentes conceptos estructurantes y formas de expresión del conocimiento, así como también su configuración, dinámicas, interdependencias mutuas e interacciones. Aunque el autor los contextualiza en la gestión tecnológica, por procesos de transferencia y analogía pueden ser aplicados a una didáctica interdisciplinaria, 
en relación con la ingeniería como disciplina fuente, integrándola a las ciencias básicas y ciencias educacionales. Desde su carácter evolutivo contribuye a la expansión adicional y progresiva del conocimiento.

De la tesis sustentada por autores como (Verloop et al., 2001; Meijer et al., 2002; Pelc, 2002; Franks y Spalding, 2013; Jablokow et al., 2015) se infiere que la cartografía conceptual permite la consolidación interdisciplinaria en espacios emergentes; considera el impacto de cada aporte de las diferentes disciplinas fuente y la afinidad entre los conceptos propios de los dominios interactuantes. Aun cuando la literatura es amplia en relación con los mapas y redes conceptuales, los referidos teóricos fundamentan la presente investigación por cuanto orientan una concepción de didáctica interdisciplinaria que fundamenta formas del pensamiento sistémico en estudiantes de ingeniería, generando capacidades de relacionamiento, ubicación espacial de conceptos, representación gráfica, así como también valorar la información y conocimiento disponible.

Autores como Meijer et al. (2002) consideran pertinente la aplicación de la cartografía conceptual para fortalecer la práctica pedagógica; describe una intencionalidad formativa. Como vía de representación permite la transferencia y compartición del conocimiento entre profesores y estudiantes; atiende su sentido y utilidad práctica desde la autorreflexión. Su carácter instrumental permite aproximarse a la producción y validación del conocimiento, durante el proceso investigativo. Constituyen herramientas gráficas con flujos relaciones de naturaleza conceptual, donde se integran experiencias, vivencias y valoraciones personales. Se infiere un tejido reticular orientador de espacios cognitivos interdisciplinarios que permita superar la fragmentación disciplinar.

La planeación didáctica en ingeniería orientada a la construcción de conocimiento de base interdisciplinaria, se valida en el contexto de las etapas del ciclo de mejora continua (PDCA: Planificar - Hacer - Verificar Actuar) propuesto por Shewhart y Deming (1939) y que mantiene su vigencia, por cuanto ayuda a la comprensión de la gestión de calidad en el ámbito organizacional, incluyendo la educación superior y más concretamente la gestión instruccional. Específicamente, se considera pertinente la incorporación del mapeo de conocimiento en el hacer didáctico, concibiéndolo como estrategia para orientar las mejoras requeridas y propuestas. La aplicación de la referida vía de producción de conocimiento interdisciplinario debe ser validada en atención a su motricidad en el sistema de relaciones, es decir cómo determina flujos, impactos, cambio y transformación en la situación problema.

\section{METODOLOGÍA}

El diseño de investigación trasciende lo descriptivo y se ubica en lo aplicativo - propositivo. Desde una visión complementaria (Martínez, 2011) se integra un componente teórico, mediante técnicas inferenciales para generar un sistema de categorías que explican en una dimensión teórica, las vías aproximativas para la didáctica interdisciplinaria de conceptos estructurantes entre ingeniería, ciencias educacionales y ciencias básicas.

Asimismo, en un plano empírico - aplicativo se validan las etapas del ciclo de mejora continua (PDCA) propuesto por Shewhart y Deming (1939) y retomados por Deming (1993), donde se configura una aproximación a un mapa de naturaleza conceptual Pelc (2002), para visualizar territorios cognitivos interdisciplinarios; específicamente se modela en la campo de la ingeniería eléctrica con el tema generador: sistema de potencia que converge en el espacio de integración con las ciencias básicas, a través de la física eléctrica y matemáticas; desde las ciencias educacionales, algunos de los dominios que entran en interacción corresponden a la didáctica, pedagogía y el currículo. El diseño de investigación es sistematizado mediante la siguiente lógica procedimental:

Se contextualiza la situación objeto de estudio en un Programa Académico de Ingeniería Eléctrica, específicamente en la asignatura curricular: Sistemas de Potencia. El objeto de estudio es la didáctica disciplinar, por su parte las unidades de análisis refieren los cursos analizados donde los sujetos están representadas por los estudiantes matriculados en el período académico 2018-02, específicamente se trabaja con un total de 40 estudiantes. Los contenidos procedimentales de la asignatura se orientan al estudio de: fundamentos conceptuales del sistema de potencia, elementos del sistema eléctrico, sistemas por unidad, flujos de cargas, análisis de fallas y contingencias, entre otros temas generadores en asociación con los específicos de las ciencias básicas.

Durante la gestión instruccional, se valida el ciclo de evolución continua: Mejora del proceso de construcción del conocimiento (enseñanza - aprendizaje) - Logro de competencias - Mediación de la estrategia didáctica Se formulan los puntos de mejora. La aplicación y validación del ciclo PDCA implica la planeación del ciclo didáctico, que toma como referente el plan de asignatura de la unidad curricular sistema de potencia. El hacer en el ciclo didáctico parte de un diagnóstico durante un periodo académico, donde la mediación didáctica se aborda de manera tradicional, privilegiando metodologías conductistas, centradas en el papel activo del docente más que del estudiante. Este diagnóstico permite identificar éxitos y fracasos en cuanto a la 
apropiación y dominio de los contenidos temáticos; se trabaja bajo la visión disciplinar de la ingeniería eléctrica y se estudian los resultados de las evaluaciones que evidencian el desempeño académico durante el 201802. Constituye el principal indicador para elaborar el plan de acción que es sistematizado de acuerdo a lo establecido en el diseño curricular de la asignatura.

El diagnóstico representa el referente para realizar los cambios en el plan propuesto e introducir la metodología sugerida, orientando las mejoras en los próximos semestres 2019-01 y 2019-02. En función del nivel de logros en el desempeño académico estudiantil, se implementa una prueba piloto a través de la enseñanza sobre sistemas de potencia y se integran los constructos propios de las ciencias básicas, específicamente la física eléctrica y matemáticas. Se produce el reforzamiento de la mediación didáctica con la incorporación de los dominios propios de las ciencias educacionales. Consecuentemente, se podría inferir un diseño orientado al cuasiexperimento (Cohen et al., 2007) por cuanto se crean las condiciones para validar el desempeño estudiantil antes y después de la intervención didáctica, a través del mapeo.

El diseño preliminar del experimento implica dos grupos con características similares, el estudio contempla toda la población de estudiantes que optaron esta asignatura durante el semestre, seleccionando de manera aleatoria un grupo como grupo control y el segundo como grupo experimental. En el primer grupo (control) se procede a trabajar de manera tradicional, con exámenes individuales y la retroalimentación respectiva, mientras que el segundo grupo (experimental) incorpora mejoras en el sistema; se presenta y explica la metodología mapeo de conocimiento en la primera sesión de trabajo del semestre; se presentan modelos de mapas para que los estudiantes los analicen en atención a los diferentes elementos o niveles conceptuales; se mantienen los exámenes individuales y la retroalimentación respectiva; conservándose los mismas pruebas o dispositivos evaluativos que pretenden validar el nivel de apropiación temática y medir el impacto que tiene en los estudiantes la estrategia interdisciplinaria en sus calificaciones finales. La principal dificultad en la ejecución de la planificación está asociada al dominio conceptual por parte de algunos estudiantes. Se trabaja con un total de 40 estudiantes, de los cuales 19 corresponden al grupo control, y 21 al experimental. Se utiliza el software $R$, para testear la normalidad de las calificaciones discriminadas según su población de procedencia, se aplica el test de normalidad de Shapiro-Wilks (Shapiro y Wilk, 1965) para ambas muestras.

Para el control y verificación se fundamenta el plan de mejora en evaluaciones individuales, minimizando los sesgos de las actividades colectivas; en estas evaluaciones se aborda la resolución de problemas estándar para estudiantes de este nivel formativo, donde por medio del uso del número de identificación del estudiante se establecen los valores de las diferentes variables. Dichos tests son aplicados en semestres sucesivos. Los resultados se ajustan al dominio cognitivo y procedimental de cada estudiante. Se retroalimentan las actividades evaluativas destacando aspectos a mejorar. En la fase de actuar, una vez finalizado el período de prueba se estudian los resultados alcanzados para luego comparar con el desempeño académico, antes de implantar la mediación didáctica con el mapeo de conocimiento. La efectividad de la metodología está guiada por las actividades pedagógicas propuestas en cada acción de mejora.

El hacer didáctico incluye la aproximación a la construcción de un mapa conceptual de conocimiento de base interdisciplinaria como experiencia de aprendizaje; toma como referente la sistematización propuesta por Pelc (2002). Al respecto, se identifican las disciplinas fuente para el conocimiento de la temática trabajada (revisión de literatura, interacción entre expertos, visión compartida entre los sujetos), de allí derivan los principales conceptos estructurantes a incluir en el mapa (dominios, contextos, categorías, constructos), para evaluar la cantidad, calidad y pertinencia de la información disponible. Se precisa el nivel de conocimiento disponible para determinar su ubicación en el mapa (realidad - representación de la realidad - modelo con referente empírico - teoría): criterios de disposición gráfica (visual, flujos, relaciones e impacto). Como técnica de procesamiento de la información se emplea el análisis e inferencia a partir de los hallazgos sistematizados. La validación metodológica se fundamenta en la triangulación subjetiva entre actores: sujetos, expertos, investigadores.

\section{RESULTADOS}

Al analizar los resultados obtenidos en el proceso evaluativo del semestre 2018-02, se tiene como principal reto la concientización del estudiante en relación con cuáles capacidades, habilidades deben poseer al ingresar a cursar la unidad curricular sistema de potencia, de forma tal que evidencien el suficiente dominio conceptual y procedimental para la resolución de problemas asociados a los contenidos propios de la asignatura. El diagnóstico se realizó mediante la identificación de las debilidades evidenciadas en las actividades evaluativas relacionadas principalmente con el dominio conceptual y procedimental de los contenidos evaluados y registrados a través de las rúbricas del docente; los resultados reflejan la necesidad por fortalecer los dominios temáticos referidos a la física eléctrica, álgebra y álgebra lineal.

Fundamentado en el diagnóstico se reorienta la planeación didáctica correspondiente a la unidad curricular sistema de potencias, percibiendo la necesidad de procesos de enseñanza globalizadores que orienten la construcción de un conocimiento interdisciplinario mediante una actividad previa, ejercicio o quiz, que permita 
a cada estudiante reconocer su necesidad de profundizar el análisis, referido tanto al temario como a la metodología. En este sentido, se determina la pertinencia conceptual y metodológica de los mapas de conocimiento para la enseñanza integrada de la ingeniería eléctrica en relación con las ciencias básicas y las ciencias educacionales, que sirva como un fundamento pragmático para la identificación de los conocimientos necesarios que permitan afrontar los contenidos de la asignatura.

Al respecto la mencionada estrategia contribuye por un lado a identificar los conceptos estructurantes de entrada que se requieren para comprender los sistemas de potencia como temática generadora, y por el otro conlleva a visualizar el sistema de flujos relacionales entre los referidos constructos; este diagnóstico conduce a reconocer debilidades en el manejo y apropiación de categorías propias de las ciencias básicas, destacando de la física eléctrica los postulados de la ley de Ohm y de Kirchhoff; del álgebra lo referido a los números complejos, y en el álgebra lineal el tema de las matrices. Desde el diagnóstico se concibe al mapeo de conocimiento como una estrategia que permite un autoreconocimiento del grado de comprensión que tiene el estudiante de los temas generadores, sus conceptos estructurantes y sistema de interacciones; así mismo para el docente como mediador didáctico representa una guía para proponer rutas de aprendizaje y fortalecer su práctica pedagógica. El mapa de conocimiento de tipo conceptual referido al tema generador sistema de potencia, que se trabaja como parte de la mejora propuesta, se observa en la Figura 1.

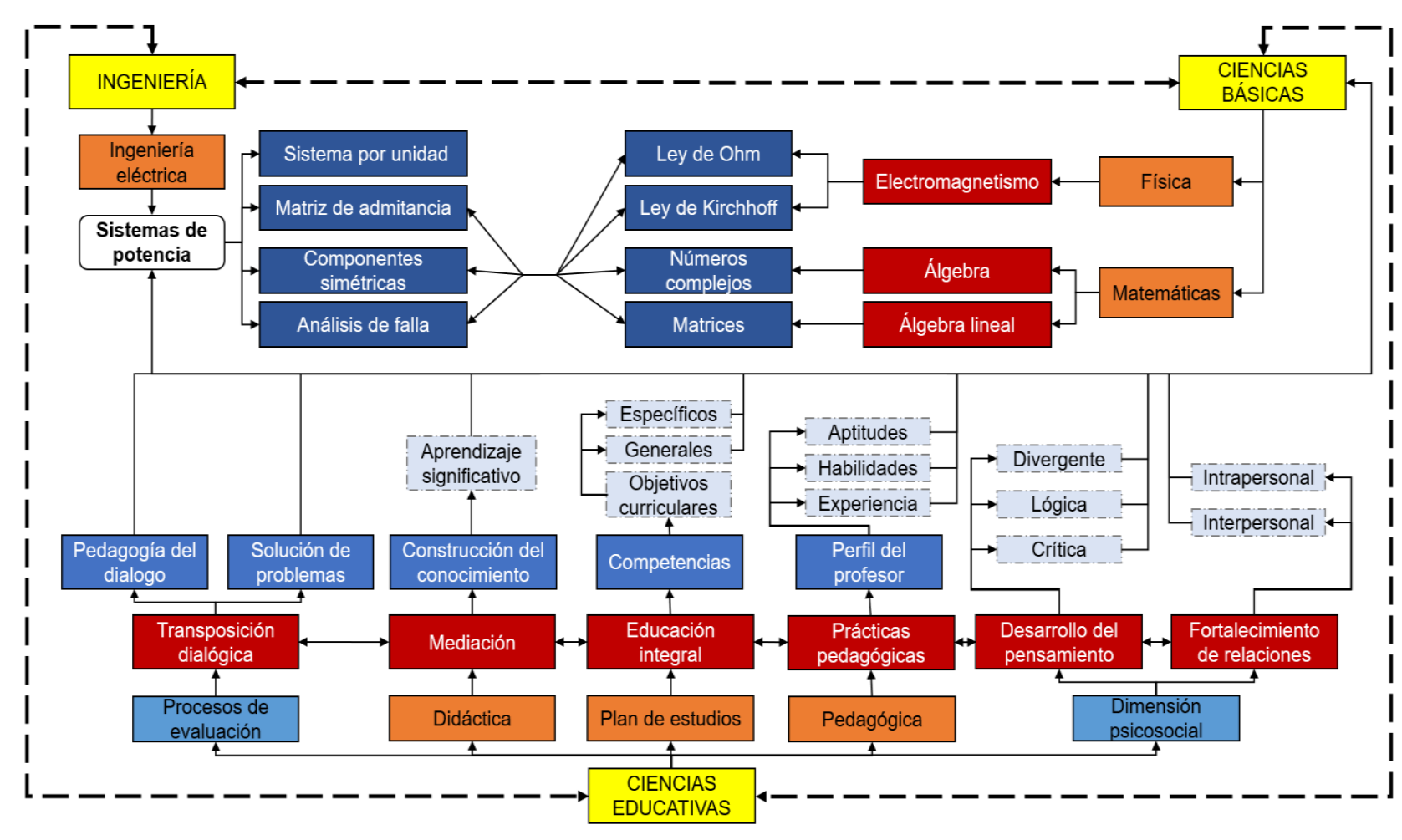

Fig. 1: Mapa de Conocimiento de Sistemas de Potencia. Elaboración de los estudiantes del grupo experimental

Para la configuración del mapa de conocimiento en la asignatura sistema de potencias, se considera el plan de asignatura e identifican los contenidos programáticos trabajados durante el trascurso del semestre; el progreso en el ciclo didáctico permite reconocer debilidades, para generar la debida retroalimentación; se avanza en la consolidación de la herramienta al integrar los conceptos propios de las ciencias básicas. Todo el proceso está soportado en aportes de los dominios educacionales, como la didáctica, currículo y pedagogía, para visualizar el amplio espectro de posibilidades que en el ejercicio educativo de la ingeniería tiene el mapeo. Su gestión implica la validación en un plano empírico de conceptos, como construcción de conocimiento, transposición didáctica, mediación didáctica, práctica pedagógica, aprendizaje significativo, perfil por competencias, formación integral, desarrollo del pensamiento, entre otros.

El diseño preliminar de la prueba piloto para la conformación del grupo control y grupo experimental permite registrar cambios, así como determinar el impacto que, en el desempeño académico estudiantil, tiene la incorporación de estrategias de mediación didácticas orientadas a la configuración de mapas de conocimiento de base interdisciplinaria. Al utilizar el software $R$ y testear la normalidad de las calificaciones discriminadas según población de procedencia, se aplica el test de Shapiro - Wilk (Shapiro y Wilk, 1965) para ambos grupos, y de allí se obtienen los resultados observados en la Tabla 1. 
Tabla 1: Test de Shapiro-Wilks para las dos muestras

\begin{tabular}{|l|l|l|l|}
\hline Grupo & Número de observaciones & Estadístico $W$ & $p$-valor \\
\hline Control & 19 & 0.93 & 0.4252 \\
\hline Experimental & 21 & 0.91 & 0.1447 \\
\hline
\end{tabular}

En función de los resultados observados en la tabla 1 se evidencia que en ambos grupos las calificaciones obtenidas tienen distribución normal. El p-valor para el grupo control es de 0.4252 y el del experimental un pvalor 0.1447 . Es decir que en ambos casos se acepta la hipótesis nula de normalidad. Con este resultado se procede a aplicar el test-t de diferencia de medias para comparar el rendimiento entre ambos grupos; los resultados se identifican en la Tabla 2.

Tabla 2: Test t de diferencia de medias independientes para las calificaciones entre el grupo control y el experimental

\begin{tabular}{|l|l|l|l|l|l|l|l|}
\hline Grupo 1 & Grupo 2 & $\begin{array}{l}\text { Media } \\
\text { grupo 1 }\end{array}$ & $\begin{array}{l}\text { Media } \\
\text { grupo 2 }\end{array}$ & $\begin{array}{l}\text { Intervalo de confianza } \\
\text { diferencia de las medias }\end{array}$ & $\begin{array}{l}\text { ¿Varianzas } \\
\text { iguales? }\end{array}$ & $\begin{array}{l}\text { Estadístico } \\
t\end{array}$ & $P$-valor \\
\hline Control & Experimental & 1.94 & 2.81 & {$[-1.58 ;-0.15]$} & No & -2.47 & 0.02 \\
\hline
\end{tabular}

Se puede inferir que la metodología es un factor que influye en las calificaciones entre ambos grupos ( $p$-valor $=0.02$ ), además se observa que el grupo experimental obtuvo unas mejores calificaciones cuyos puntajes superan entre 0.15 y 1.58 a las calificaciones del control; se infieren diferencias significativas entre ambos grupos, lo que permite denotar la efectividad del mapeo como estrategia didáctica, que permite la construcción de un conocimiento de base interdisciplinaria entre ingeniería, ciencias básicas y educación para mejorar el desempeño académico evidenciado en un mejoramiento significativo en las calificaciones obtenidas por los participantes.

\section{DISCUSIÓN}

Los mapas de conocimiento representan una metodología válida y pertinente para la enseñanza integrada e interdisciplinaria de conceptos estructurantes entre el área de ingeniería y disciplinas complementarias, como educación y ciencias básicas. La sistematización de experiencias en atención al surgimiento de territorios cognitivos interdisciplinarios permite visualizar cómo los conceptos estructurantes, expresados en teorías, constructos, estrategias, técnicas de trabajo, propios de cada disciplina interactuante se integran desde un sentido convergente, para consolidar un sistema reticular relacional de naturaleza hibrida, cuyo énfasis está centrado en el conocimiento que se genera para explicar la situación o temática estudiada.

El mapeo de conocimiento como vía heurística para una didáctica interdisciplinaria, contribuye a un diagnóstico por parte del docente acerca de las competencias cognitivas y procedimentales que desarrollan los estudiantes, para la integración de conceptos estructurantes entre ingeniería, educación y ciencias básicas; igualmente permite que los estudiantes se hagan conscientes de sus capacidades para apropiarse de conceptos y procesos, de manera tal que pueda comprender la lógica relacional y los significados atribuidos a dichas relaciones con base a sus vivencias, experiencias y rutinas. Los estudiantes mediante la representación gráfica confieren significados a la red de relaciones conceptuales emergente, y para el docente representa una guía de los diferentes itinerarios de mediación para la construcción de un conocimiento integrador.

\section{CONCLUSIONES}

De acuerdo al trabajo presentado y a los resultados obtenidos, se pueden plantear las siguientes conclusiones principales:

1. Los estudiantes evidencian mejoras en su rendimiento académico cuando emplean los mapas de conocimiento para integrar contenidos relacionados propios de las disciplinas interactuantes

2. La aplicación de la metodología permite la integración de saberes disciplinares y pedagógicos con la debida retroalimentación en función de los resultados.

3. La aplicación de estos procesos didácticos en otras asignaturas y en condiciones definidas contribuirá a validar su efectividad y pertinencia. 


\section{REFERENCIAS}

Albert-Gómez, M. J., García-Pérez, M. y Pérez-Molina, C., Competencias, Formación y Empleo. Análisis de Necesidades en un Programa de Master en Ingeniería. http://dx.doi.org/10.4067/S0718-50062017000200006. Formación universitaria 10(2), 43-56 (2017).

Ausubel, D., Teoría del aprendizaje significativo, Fascículos de CEIF, (1983).

Bravo, O. Marín-González, F. y Carrera, M., Redes Interorganizacionales y Desarrollo Local, OPCIÓN 29(70), 86-103 (2013).

Cabero, J., y Barroso, J., ICT teacher training: a view of the TPACK model / Formación del profesorado en TIC: una visión del modelo TPACK. https://doi.org/10.1080/11356405.2016.1203526. Cultura y Educación, 28(3), 633-663 (2016).

Castro Superfine, A., y Li, W., Exploring the Mathematical Knowledge Needed for Teaching Teachers. https://doi.org/10.1177/0022487114534265 Journal of Teacher Education, 65(4), 303-314 (2014).

Cervantes Loredo, M. T., García Quintanilla, M., y Cuevas Gutiérrez, A. I., El trabajo interdisciplinario en una red académica: desafío y complejidad, Ciencia UANL, 19(79), 40-44 (2016).

Chevallard, Y., La transposition didactique, Vol. 95, (1985).

Cohen, L., Manion L., Morrison K ., Research methods in education. Sixth Edition. Routledge of Taylor and Francis. New York. (2007)

Coll, C., y Engel, A., Introduction: Personal Learning Environments in the context of formal education / Introducción: los Entornos Personales de Aprendizaje en contextos de educación formal. https://doi.org/10.1080/11356405.2014.985947. Cultura y Educación, 26(4), 617-630 (2014).

De Bono, E., El pensamiento práctico. Barcelona, España (1992).

Deming, W. E., The New Economics: for industry, government, education, Cambridge: MIT press (1993).

Domínguez Osuna, P.M., Oliveros Ruiz, M.A., Coronado Ortega, M.A., y Valdez Salas, B., Retos de ingeniería: enfoque educativo STEM+A en la revolución industrial 4.0. Innovación educativa (México, DF), 19(80), 15-32 (2019).

Follari, R., La interdisciplina en la docencia, POLIS, Revista Latinoamericana, 6(16), 1-12 (2007).

Espinoza, R., y Marín-González, F., Redes de investigación transdisciplinar tecnocientífico en contextos reticulares. https://dx.doi.org/10.4067/S0718-50062018000100013. Utopía y Praxis Latinoamericana, [S.I.], 24(87), 173-193 (2019).

Franks, R. A., y Spalding, A. D., Business ethics as an accreditation requirement: A knowledge mapping approach, Business Education \& Accreditation, 5(1), 17-30 (2013).

Guzmán, I., Settati, A., y Marín, R., Transdisciplinariedad y la transversalidad. https://doi.org/10.17981/cultedusoc.10.2.2019.06. Cultura Educación y Sociedad, 10(2), 73-84 (2019).

Jablokow, K. W., DeFranco, J. F., Richmond, S. S., Piovoso, M. J., y Bilén, S. G., Cognitive Style and Concept Mapping Performance, Journal of Engineering Education, 104(3), 303-325 (2015).

Kennedy, T. J, y Odell, M. R. L., Engaging students in STEM education, Science Education International, 25(3), $246-258$. (2014)

Marín-González, F.; Cabas, L.; Cabas, L. y Paredes-Chacín, A., Formación Integral en Profesionales de la Ingeniería. Análisis en el Plano de la Calidad Educativa. http://dx.doi.org/10.4067/S0718-50062018000100013. Formación universitaria, 11(1), 13-24 (2018).

Martínez Miguélez, M., Paradigmas emergentes y ciencias de la complejidad, Opción, 65(45-80), 27 (2011).

Meijer, P. C., Zanting, A., y Verloop, N., How can student teachers elicit experienced teachers' practical knowledge? Tools, suggestions, and significance. https://doi.org/10.1177/002248702237395 Journal of Teacher education, 52(5), 406-419 (2002).

Palomares Montero, D., y Chisvert Tarazona, M. J., Cooperative learning: a methodological innovation in teacher training I El aprendizaje cooperativo: una innovación metodológica en la formación del profesorado. https://doi.org/10.1080/11356405.2016.1158448. Cultura y Educación, 28(2), 378-395 (2016).

Pelc, K. I., Knowledge mapping: The consolidation of the technology management discipline. https://doi.org/10.1007/s12130-002-1003-3. Knowledge, Technology \& Policy, 15(3), 36-44 (2002).

Piaget, J., Piaget's theory in PH Mussen (1970).

Quarless, D., y Nieto, F., Exploring Hybrid Instruction in Science: Using LMS for Contextual, Interdisciplinary Active Learning Enrichment. https://doi.org/10.2190/ET.41.3.f. Journal of Educational Technology Systems, 41(3), 279-292 (2013).

Ramirez, C. C., Zartha, J. W., Arango, B., y Orozco, G. L., Prospectiva 2025 de la Carrera de Ingeniería Química en algunos Países pertenecientes a la Organización de Estados Americanos (OEA). http://dx.doi.org/10.4067/S071850062016000600012. Formación universitaria, 9(6), 127-138 (2016). 
Robles-Fernández, A., Pedrosa, I., Suárez-Álvarez, J., Blanco-Saldaña, I., y García-Cueto, E., Curricular change and educational quality / Cambio de plan y calidad educativa. https://doi.org/10.1080/11356405.2016.1269503. Cultura y Educación, 29(1), 97-119 (2017).

Rodríguez, M., La interdisciplinariedad: Acción comunicativa científica y humana, Universidad de Antioquia. (2009)

Shapiro, S. S., y Wilk, M. B., An Analysis of Variance Test for Normality (Complete Samples). https://doi.org/10.2307/2333709. Biometrika, 52(3/4), 591-611 (1965).

Shewhart, W. A., y Deming, W. E., Statistical Method from the Viewpoint of Quality Control, Courier Corporation (1939).

Steffens, E., Ojeda, D., Martínez,O., García, J., Hernández, H., Marín, F., Niveles de pensamiento crítico en estudiantes de Universidades en Barranquilla (Colombia), Espacios, 38(30), 5-15. 2017

Tobón, S., Competencias en la educación superior: políticas hacia la calidad. Bogotá, Colombia (2006).

UNESCO, Los jóvenes y las competencias: Trabajar con la educación. Ediciones UNESCO Segunda edición (2012).

Van der Linde, G., ¿Por qué es importante la interdisciplinariedad en la educación superior?. https://doi.org/10.29197/cpu.v4i8.68. Cuadernos de Pedagogía Universitaria, 4(8), 11-13 (2007).

Verloop, N., Van Driel, J., y Meijer, P., Teacher knowledge and the knowledge base of teaching. https://doi.org/10.1016/S0883-0355(02)00003-4. International Journal of Educational Research, 35(5), 441-461 (2001).

Vygotsky, L. S., The collected works of LS Vygotsky: Problems of the theory and history of psychology. Vol 3. Springer Science \& Business Media (1997). 